\title{
Research on the precision information service for agricultural technology extension based on situational awareness
}

\author{
SUN Xiang ${ }^{1,2,3,4, a}$, WU Huarui ${ }^{1,2,3,4, b_{*}}$, LI Qingxue ${ }^{1,2,3,4, c}$, HAO Peng ${ }^{1,2,3,4, d}$ \\ ${ }^{1}$ Beijing Research Center for Information Technology in Agriculture, Beijing Academy of Agriculture \\ and Forestry Sciences, Beijing, 100097, China \\ ${ }^{2}$ National Engineering Research Center for Information Technology in Agriculture, Beijing 100097, \\ China \\ ${ }^{3}$ Key Laboratory of Agri-informatics, Ministry of Agriculture, Beijing 100097, China \\ ${ }^{4}$ Beijing Engineering Research Center of Agricultural Internet on Things, Beijing 100097, China \\ a'sunx@nercita.org.cn, bwuhr@nercita.org.cn(*corresponding author), 'liqx@nercita.org.cn, \\ dhaop@nercita.org.cn
}

Keywords: agricultural technology extension, situational awareness, precision information service

\begin{abstract}
Scenario aware computing is an important technology to realize precise information service in agricultural technology extension. This paper constructs a scene data fusion user perception of precision agricultural extension service. This paper established user classification model and multi situational awareness recommendation model based on multiple-factor, and realized the intelligent push services in personalized information. In comparison to common retrieval system which did not have the situational awareness and the intelligent push functions, the personalized information services based on situational awareness calculation was able to identify and predict the preferences of user interest, provided the psychological content for users. The correlation of personalized information services and resource push based on situational awareness calculation increased on average by 2.22 times, the accuracy rate increased by 1.34 times, meeting the user's personalized needs.
\end{abstract}

\section{Introduction}

In recent years, the research of situational awareness technology has developed rapidly at home and abroad. Situational awareness and networking, intelligent processing, mobile Internet and other technologies are closely related, to be able to take the environment as the guidance, the keen perception scene, and give the adaptation response [1-3]. In agriculture, some scholars have proposed the use of context-aware computing technology to improve agricultural networking comprehensive efficiency in precision agriculture production, agricultural product logistics, agricultural marketing and agricultural services, and other major efficiency [4]. Agricultural extension services mainly for farmers, rural area development level is relatively backward, according to user characteristics, information needs and behavior to provide targeted and accurate information service information service is to solve the "last mile" problem of the effective technical means [5].

This paper aims to construct a precise agricultural extension services mode, that can perceive the agricultural production environment, crop growth status, behavior management and other production site data, combined with reasonable categories of users, the user is interested in agricultural knowledge timely, accurate pushed to farmers, promoting agricultural operations to maximize efficiency and rationalization, research results in the field of agriculture to play its due role.

\section{Classification of Situational Awareness Information}

Situational awareness information generally includes the following levels (Figure 1), including environmental scenarios, user scenarios, and operational scenarios.

Environmental scenarios includes farm location, area, soil conditions, varieties, tree-age, growth 
period, pests, fertilizer and other information[6-7]; user scenarios including user types, user preferences, user needs, the user region and age; operating scenarios including interactive behavior, system function access probability of information services and others.

In the process of agricultural information services, different regions, different time, different information needs of individuals vary, most of these differences can be embodied by environmental scenarios. As in the south orchards, growers are interested about the information of tangerine, mango and other south fruit varieties, the north growers are interested about the information of peach, jujube, apricots and other varieties; agriculture technical staff like text information, and farmers tend to the simple easy to understand information, such as pictures, videos.

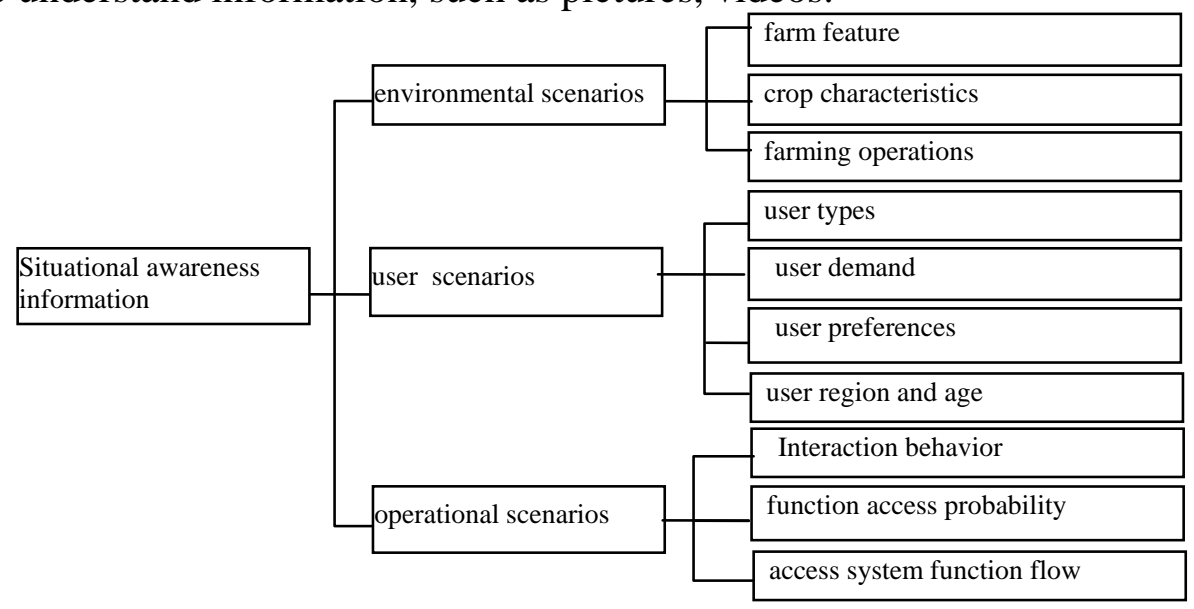

Figure 1. production management scenario classification

\section{User Classification Model and Recommendation Model}

User classification model. The user characteristic classification factor is defined as: \{geographical features $\mathrm{R}$, time characteristics $\mathrm{T}$, crop varieties $\mathrm{V}$, production and management links $\mathrm{P}\}$.These factors have a clear order, geographical features as the first factor, the time characteristics as a factor of second, the variety of characteristics as a factor of third, production and operation links as a fourth factor. According to the order of the 4 factors, the order of the relationship is gradually divided into different user sets, as shown in figure 2.

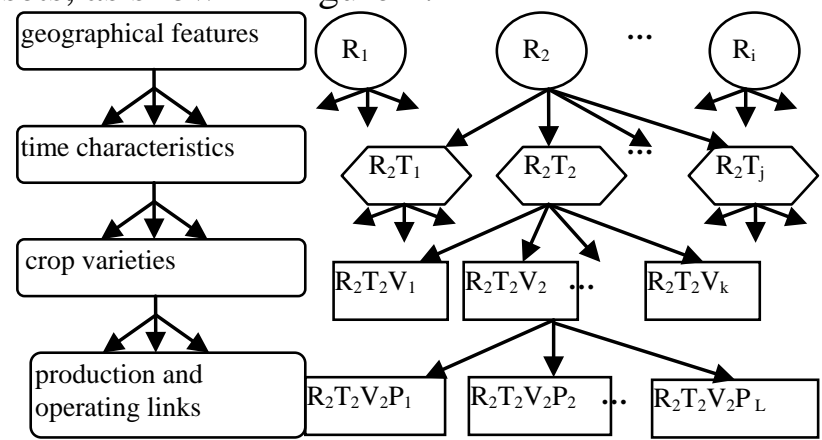

Figure 2. $\quad$ User classification

In addition, Agriculture production and operation links can be divided as shown in Table 1.

Table 1 Division of vineyard production and management

\begin{tabular}{|c|c|c|}
\hline crop varieties (V) & production and operation links (P) & interest feature words (C) \\
\hline \multirow{5}{*}{ grape } & production management & $\begin{array}{l}\text { Grape cultivation, pest control, nutrition diagnosis, } \\
\text { vineyard plan }\end{array}$ \\
\hline & agricultural material supply & Fertilizers, pesticides, seeds, mulch, etc. \\
\hline & fruit circulation & $\begin{array}{l}\text { market price, supply and demand information, } \\
\text { brokers, etc. }\end{array}$ \\
\hline & fruit processing & fruit juice, wine, raisins, etc. \\
\hline & information service & $\begin{array}{l}\text { agricultural super docking, the surrounding wine } \\
\text { factory, etc. }\end{array}$ \\
\hline
\end{tabular}




\section{User interesting model.}

The user interest model is based on the user classification model, which can be expressed as the interest factor and the degree of interest:

$\mathrm{N}=\alpha R_{i}+\beta T_{j}+\gamma V_{k}+\delta P_{L}$

The geographical feature vector is expressed as:

$R_{i}=\left\{\left(R_{1}, w_{1}\right),\left(R_{2}, w_{2}\right), \ldots,\left(R_{i}, w_{i}\right)\right\}$

Where $\mathrm{i}$ is the number of regional interest factors, the m number of factor is $\mathrm{Rm}, w_{1}$ is the weight of the $\mathrm{m}$ number of factor, and $\sum_{m=1}^{i} R_{m}=1$.

The user time characteristic vector is expressed as:

$T_{j}=\left\{\left(T_{1}, w_{1}\right),\left(T_{2}, w_{2}\right), \ldots,\left(T_{j}, w_{j}\right)\right\}$

Where $\mathrm{j}$ is the number of time characteristic vector, the m number of factor is $T_{m}, w_{1}$ is the weight of the m number of factor, and $\sum_{m=1}^{j} T_{m}=1$

The variety characteristic vector is expressed as:

$V_{k}=\left\{\left(V_{1}, w_{1}\right),\left(V_{2}, w_{2}\right), \ldots,\left(V_{k}, w_{k}\right)\right\}$

Where $\mathrm{k}$ is the number of time characteristic vector, the m number of factor is $V_{m}, w_{1}$ is the weight of the m number of factor, and $\sum_{m=1}^{j} V_{j}=1$

The production and operation links vector is expressed as:

$P_{L}=\left\{\left(P_{1}, w_{1}\right),\left(P_{2}, w_{2}\right), \ldots,\left(P_{L}, w_{L}\right)\right\}$

Where $\mathrm{L}$ is the number of time characteristic vector, the m number of factor is $P_{m}, w_{1}$ is the weight of the m number of factor, and $\sum_{m=1}^{L} P_{L}=1$

Each interest factor can be expressed by $\mathrm{N}$ feature words; the interest vector of feature words is expressed as:

$$
\begin{aligned}
& \mathrm{R}_{p}=R_{p}\left(r_{1}^{1}, r_{1}^{2} \ldots, r_{1}^{n}\right), \mathrm{p} \in[1, \mathrm{i}] \\
& \mathrm{T}_{p}=T_{p}\left(t_{1}^{1}, t_{1}^{2} \ldots, t_{1}^{n}\right), \mathrm{p} \in[1, \mathrm{j}] \\
& \mathrm{V}_{p}=V_{p}\left(t_{1}^{1}, t_{1}^{2} \ldots, t_{1}^{n}\right), \mathrm{p} \in[1, \mathrm{k}] \\
& \mathrm{P}_{p}=P_{p}\left(t_{1}^{1}, t_{1}^{2} \ldots, t_{1}^{n}\right), \mathrm{p} \in[1, \mathrm{~L}]
\end{aligned}
$$

\section{Agricultural Information Intelligent Push}

The information recommendation model consists of two stages(Figure 3).

\section{User feature description}

First, the perception of the information noise processing, filter out the system noise. Then according to the user's context aware information were feature extraction, user interest feature vector set is established, to determine the weights of factors, and according to the tracking and analysis of user behavior and constantly revised user interest feature vectors. Finally, the user interest is expressed, to achieve the description of user characteristics [8].

- In the process of situational information perception, users can also determine the geographical features of the user's IP address, phone number, thus narrowing the scope of the recommended information, improve the pertinence of the recommended information.

- The production of orchard has the characteristics of seasonal and cyclical. In different seasons, the growth period of fruit trees is different, so the information that the user is interested in each time period will also be different. According to the growth stage of the fruit trees in the area where the user is located, the scope of the recommended information is reduced, and the pertinence of the recommended information is improved. 


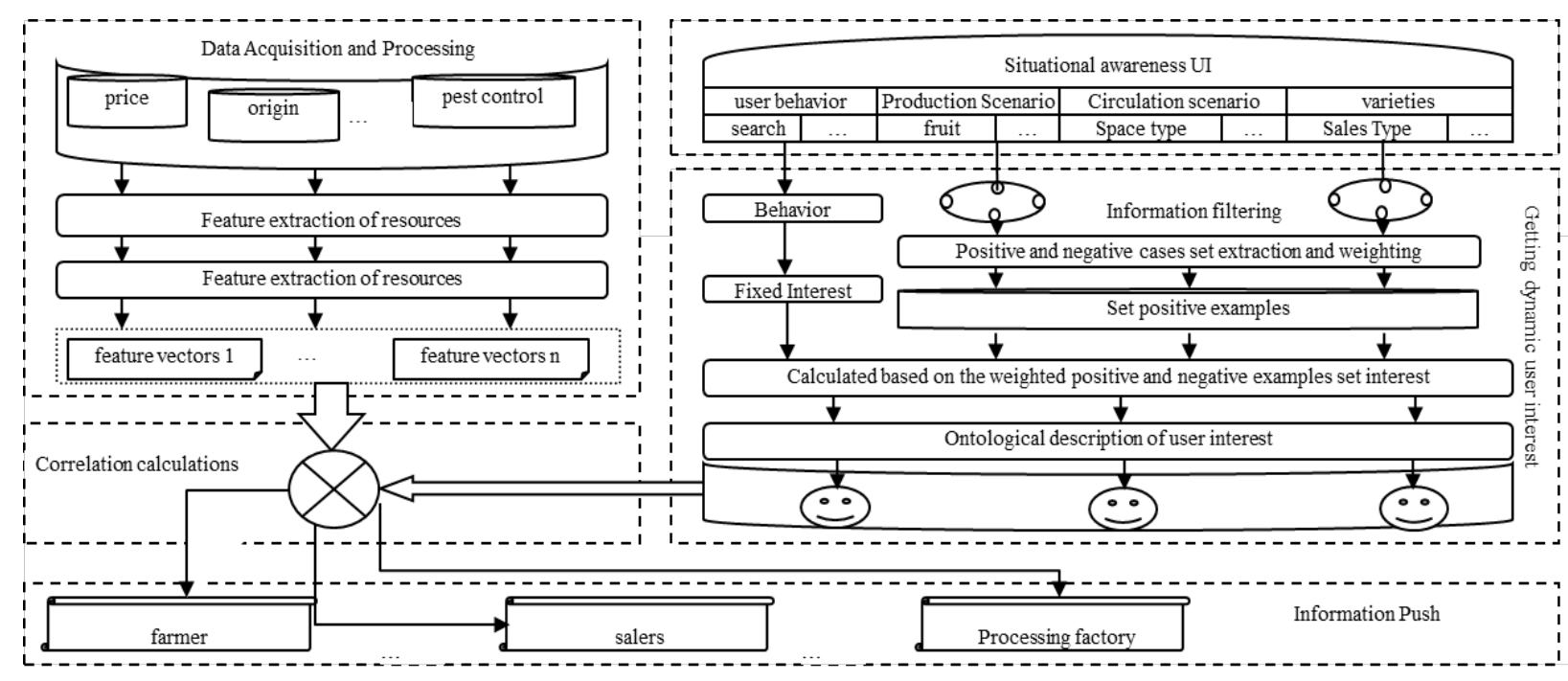

Figure 3. Context aware and personalized information intelligent push process

\section{Feature vector description of resources}

All kinds of information resources according to the user classification model were feature extraction, establishment of resource description framework, and in accordance with the expansion of the concept of the characteristics of polysemy, synonymy, hyponymy, realizes the feature vectors to specific information resource description.

\section{Information resources matching calculation}

Calculate the similarity of information resources characteristic vector and user interest vector, according to the similarity of the results established the recommendation and user needs related to the similar, synonymy or nearly righteousness, access history and hotspot access set, matching the information needs of users and information sources.

If there is a perceived blind lead to recognition and matching information in the process of pushing, based on semantic memory of pushing information to improve the characteristics of the information resource for the vector and the user interest vector similarity matching degree.

\section{Experimental Results and Analysis}

In this paper, the personalized information service model is integrated into the information service platform. In order to verify the effectiveness of the model, this paper compares it with the personalized push system based on key words:

\section{Experimental data}

Include different types of fruit production and management stage of the 2000 resource content, including the 12 types of interest factors, about 120 interest feature words.

\section{Experimental user}

The user is FangShan district viticulture farmer, who is concerning the market price of grapes, viticulture, supporting disease control, plant protection experts.

\section{Experimental operation}

a) The system records the log information of the user operation behavior. The user profile information to provide the next analysis.

b) To enter the user "personality push" module, access to its list of recommended resources.

c) Close the "User interest model" function, the same user Repeat steps (a), (b) operation obtains its list of recommended resources.

The results of the experiment are shown in table 2. The analysis is carried out according to the experimental data, 10 cycles of operation results can be seen that the correlation of personalized information services and resource push based on situational awareness calculation increased on average by 2.22 times, the accuracy rate increased by 1.34 times. 
Therefore, the personalized information services technology based on situational awareness can provide users with targeted services, and to meet the needs of users.

Table 2 Results of test

\begin{tabular}{|l|l|l|l|l|l|l|l|l|l|}
\hline \multirow{2}{*}{ Id } & \multicolumn{2}{|c|}{$\mathbf{A}$} & \multicolumn{2}{c|}{ Ra } & \multicolumn{2}{c|}{ Ra/A } & Rb & \multicolumn{2}{c|}{ Ra/Rb } \\
\cline { 2 - 10 } & $\boldsymbol{I}$ & $\boldsymbol{K}$ & $\boldsymbol{I}$ & $\boldsymbol{K}$ & $\boldsymbol{I}$ & $\boldsymbol{K}$ & $/$ & $\boldsymbol{I}$ & $\boldsymbol{K}$ \\
\hline 1 & 56 & 59 & 48 & 31 & 85.71 & 52.54 & 55 & 87.27 & 35.52 \\
\hline 2 & 45 & 41 & 35 & 23 & 77.78 & 56.10 & 42 & 83.33 & 27.60 \\
\hline 3 & 33 & 34 & 27 & 22 & 81.82 & 64.71 & 35 & 77.14 & 28.52 \\
\hline 4 & 35 & 37 & 34 & 26 & 97.14 & 70.27 & 45 & 75.56 & 34.41 \\
\hline 5 & 61 & 72 & 52 & 34 & 85.25 & 47.22 & 67 & 77.61 & 43.81 \\
\hline 6 & 66 & 77 & 58 & 45 & 87.88 & 58.44 & 68 & 85.29 & 52.76 \\
\hline 7 & 64 & 54 & 58 & 34 & 90.63 & 62.96 & 65 & 89.23 & 38.10 \\
\hline 8 & 40 & 41 & 34 & 32 & 85.00 & 78.05 & 45 & 75.56 & 42.35 \\
\hline 9 & 36 & 37 & 30 & 28 & 83.33 & 75.68 & 40 & 75.00 & 37.33 \\
\hline 10 & 38 & 24 & 32 & 18 & 84.21 & 75.00 & 40 & 80.00 & 22.50 \\
\hline
\end{tabular}

Note: the "I" represents the result of intelligent information push method, the " $\mathrm{K}$ " represents the result of the Key word information push method; the " $A$ " represents the number of resources retrieved in the table, "Ra" represents the number of relevant resources retrieved, "Ra/A" is precision, and "Rb" represents the number of all relevant resources

\section{Conclusions}

Accurate information services has become an important part of the current agricultural extension, this article will focus on how agriculture production site scenarios reasonable definition and classification in order to achieve accurate and personalized information services for content research, including agricultural production site scenario definition, based on more user classification model factor, user interest model and intelligent push personalized information and other content, and experimental analysis, recommendations are based on intelligent user interest model in a push resource dependency is obviously superior to conventional methods of information push, push relevant resources of the average increase on average by 2.22 times, the accuracy rate increased by 1.34 times, to meet the individual needs of users. With the gradual rise of situational awareness technology in artificial intelligence, and agricultural extension services around the different characteristics of application scenarios, further targeted research to build models and classify user interest model is the focus of future research applications.

\section{Acknowledgement}

This work was supported by Special Fund for Agro-scientific Research in the Public Interest (201303107).

\section{References}

[1] Gu Junzhong.Context aware computing[J]. Journal of East China Normal University: Natural Science, 2009 (5): 1-20

[2] Schilit B, Adams N, Want R. Context-Aware Computing Applications[C]// The Workshop on Mobile Computing Systems \& Applications. IEEE Computer Society, 1994:85--90.

[3] Schirmer M, Bertel S, Pencke J. The Contexto Framework: Leveraging Energy Awareness in the Development of Context-Aware Applications[M]// Advances and New Trends in Environmental and Energy Informatics. Springer International Publishing, 2016:9512-9519.

[4] Yang Baozhu, Liu Yan, Wu Huarui,Zhu Huaji. Personalization Agriculture Information Service Model Based on Context-aware Computing [J]. Agricultural Mechanization Research, 2012 (11): 10-15 
[5] Xiao Boxiang, Guo Xinyu, Wang Chuanyu,Wu Sheng, Du Jianjun,Lu Shenglian,etc. Discussion on Application of Context Aware Computing Technology in Agricultural Internet of Things [J]. Agricultural Science and technology, 2014 (5): 21-31.

[6] Ingole P, Pawar J, Vhatkar H. Context Aware, Mobile Phone Based Sink Model of Wireless Sensor Network for Indian Agriculture[M]// Advances in Computing, Communication, and Control. Springer Berlin Heidelberg, 2013:301-308.

[7] Song Bin, Chen Liping, Chen Tianen. Application Research on Context Aware Technology in Facility Agriculture Based on Internet of Things [J]. Anhui agricultural science, 2015 (20): 369-371.

[8] Chen Cheng, Liao Guiping, Shi Xiaohui, Zhao Xing. The Personalized Push Technology of Agriculture and Rural Information Service [J]. Chinese Agricultural Science Bulletin, 2011, 27 (29): 151-156. 